\title{
A User-Centered, Integrated Model to Improve Medication Prescription, Administration and Adherence in Switzerland
}

\author{
Katherine BLONDON ${ }^{\mathrm{a}, 1}$ and Frederic EHRLER ${ }^{\mathrm{b}}$ \\ ${ }^{a}$ Medical Directorate, University Hospitals of Geneva, Switzerland \\ ${ }^{\mathrm{b}}$ Direction of Information System, University Hospitals of Geneva, Switzerland
}

\begin{abstract}
Medication adherence remains an obstacle for the ideal medical care. Communication issues arise between care-providers, and the patient is left to deal with potentially conflicting information. The new electronic patient record (EPR) that will soon be implemented nationally opens new perspectives to improve patient medication management. In this context, we propose an integrated model that could help further empower the patient with better communication about medications and considerations for reconciliation processes. We discuss important considerations for our proposed solution.
\end{abstract}

Keywords. Care Network, Medication Management, EPR, Medication Adherence

\section{Introduction}

Despite the many improvements in healthcare over the past years, the patient's road from being diagnosed to receiving medical treatment and subsequent improvement remains bumpy [1]. Patients often have more than one healthcare provider, who may each prescribe medications without necessarily knowing about the patient's other prescriptions. Patients can go to any pharmacy to receive their medicine, so the pharmacist may not know about other on-going treatments [2]. Patients may even receive contradictory information about their medication from their multiple healthcare providers, leading to confusion and yet lower adherence to treatment [3]. The family doctor often receives reports from the specialists about their shared patients, allowing them to play an important role in the medication reconciliation process. Many gaps during transitions and difficulties in sharing information to collaboratively manage a patient remain present, resulting in fragmented care $[4,5]$. The current reconciliation process largely relies on the patient to communicate about their treatments to other healthcare providers.

Despite the implementation of electronic medical records in hospitals, clinics and many private practices in Switzerland, there is no secure, systematic communication channel between the various healthcare networks [6]. Patient portals also show potential

\footnotetext{
${ }^{1}$ Corresponding Author, Katherine Blondon, University Hospitals of Geneva, Gabrielle Perret Gentil, 4 1205 Geneva, Switzerland E-mail: Katherine.Blondon@hcuge.ch.
} 
in helping patients management their medications [7]. The long-awaited electronic patient record (EPR) may provide new opportunities for patient empowerment, particularly for medication management.

In this paper, we propose a user-centered model to help improve the flow of information about a patient's treatments, including the patient's self-management at home. This model is proposed based on the experience collected through several projects around medication such as the redesign of our hospital computerized order entry system, the development of an application for the daily management of patients' treatment [8], the participation to the design of the shared medication plan of the Swiss EPR [9] and a working group around medication reconciliation. All these inputs processed by the authors helped us to model the current patient journey around their medication and to identify the existing gaps. Based on the evolution of the digital landscape, especially with the introduction of the EPR, we propose an integrated model aiming to fill these gaps. We then discuss important considerations for the implementation of this model.

\section{Current Considerations and Existing Tools}

As mentioned above, there are several gaps in the communication process between the doctor's prescription, the patient's self-management and long-term adherence to treatment. In a simplified, single doctor-patient model, the doctor diagnoses a condition, then prescribes an appropriate medication: after explaining why and how to take the medicine, the doctor hands the paper with the prescription to the patient. The patient heads to the pharmacy, and receives the box of medicine with instructions and precautions on how to take the medicine. Back at home, the patient is then in charge of taking the medication regularly as prescribed. The patient needs to understand what to do, and why it should be done, which in turn should help drive motivation to follow through with the recommendations until the end of the prescribed treatment.

In a multiple-provider model with a family doctor, the family doctor could ideally help centralize the patient's medication information, and adjust and adapt treatments as needed. Practically, however, the patient is the one who goes from one provider to another: as there is yet little systematic communication between healthcare professionals, the flow of information about medications often depends on what the patient understands, recalls, and can report. It also depends on what the provider discusses with the patient.

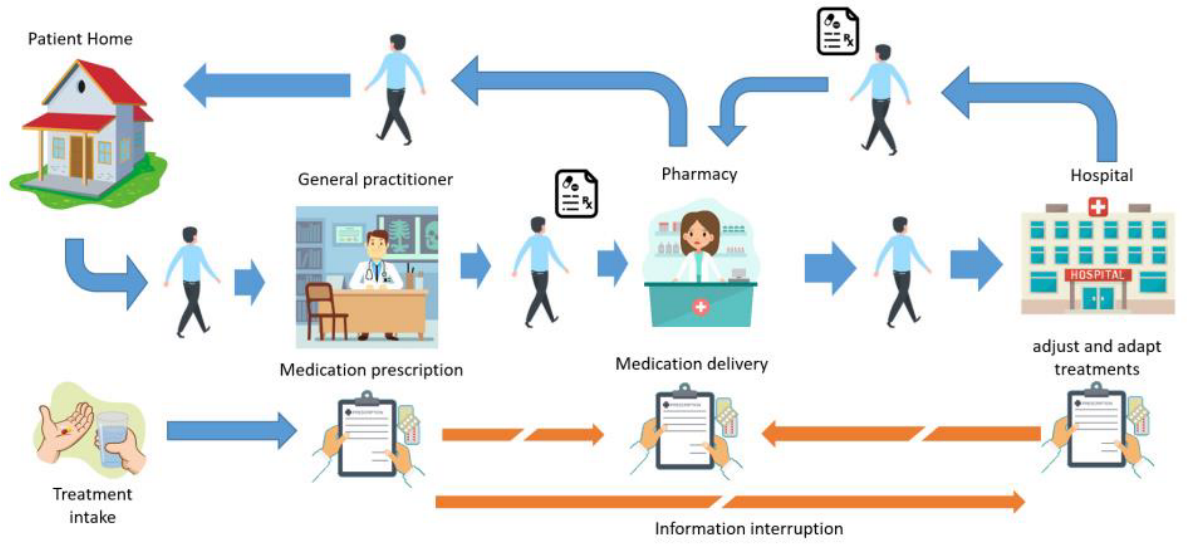


Conflicting health information is a growing concern, with increasing sources of information (e.g. digital sources [10]), in particular for medication [11]. In a recent study of 405 pharmacy customers, $47 \%$ of individuals declared that they had received some discordant information in some way about their medication (from their various healthcare providers, from TV programs or commercials, from social media or from family and friends). About two thirds subsequently changed their medication behavior.

The future national EPR record will soon be implemented, and will include a module called "shared medication plan"[12]. This electronic record aims to help allow the patient to have access to her medical information, and to share it with whoever she wishes. This medication plan module will allow doctors in various settings (private practice or institutions) to export their prescriptions to the patient's EPR. The pharmacist's system will import the prescriptions and will link them with the given medication (exact medication name, dose and amount in the package). The patient will be able to access ant interact with the medication list (such as over the counter medications). The patient can add comments to all medications, but can only edit those entered by the patient.

\section{Proposed Model}

We propose an integrated model based on the future national electronic patient record and its medication plan module, which will also be integrated with our local institution's electronic medical record.

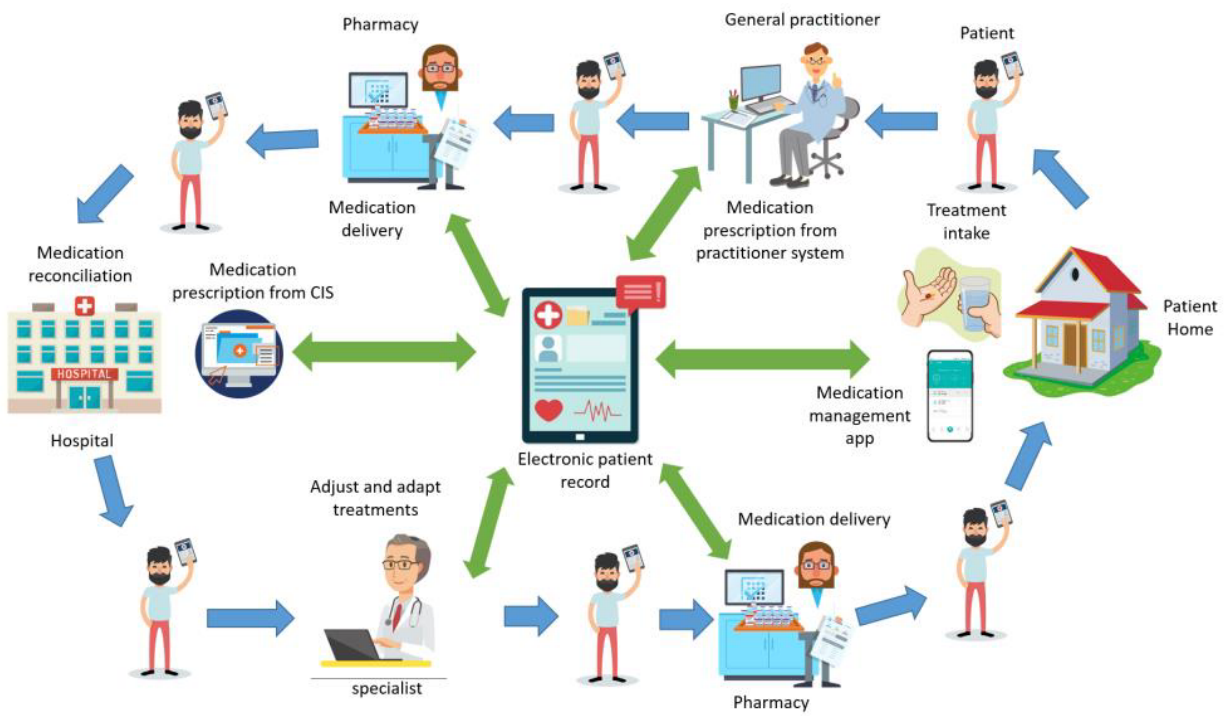

Our aim with the integrated model is to address the patient's daily needs for medication self-management and to discuss some practical considerations in the integration process. In this model, the EPR centralizes the information from the hospital and from the various care-providers: when the patient sees the family doctor, the report and medication are synchronized with the EPR. The patient goes to the pharmacy: the pharmacist can access the prescribed medications, and adds the exact package that was delivered to the patient (brand or generic, number of pills, etc.). Once the patient arrives 
at home, she can double check the medications she has (those just received, as well as any prior medications) by scanning the barcodes in the app: this creates a reconciled list that can also include any over-the-counter medications. If the patient is admitted to the hospital, the medication list from the app can be synchronized with the hospital's computerized prescription tool, with additional information from the tracking and monitoring tools if the patient has used them. At discharge from the hospital, the prescribed medication list is again updated in the EPR, and available for the pharmacist to deliver the medication.

The patient is the only person that is present at each step of his own care: he needs to have access to an updated medication list at all times, and to be able to share it with whomever he wishes at any time. One approach to empower the patient is to provide support for the medication plan, and to help them manage their medications daily with reminders and tracking tools $[13,14]$. Based on focus groups and patient interviews, with iterative testing and improvements, a medication app called Swiss-meds was developed to provide these functionalities[15]. Patients discharged from the hospital often receive a list of new medications, and one difficulty that the project participants reported was not knowing which medicines to take, between the new prescriptions and the medication that their family doctor had prescribed before the hospital stay. This app therefore also included an alert for when similar medications are entered in the medication plan, suggesting that the patient ask the pharmacist or doctor about these potential duplicates. Another request for those who track their medicine was to be alerted in time to fetch a new box of medication before running out. Furthermore, low-literacy information sheets and links to the official patient information website in the app allow the patients to learn about their medication.

\section{Implications of an App for an Integrated Solution}

The medication app for the patient addresses self-management needs, but can also help provide links between the patient and the other actors in the model: besides empowering the patient with information about the medications, it allows the patient to share the collected data with the care-providers. In an integrated model between the patient and the healthcare system, the medication list from the electronic medication plan, enriched with the actual medication packages received at the pharmacy, should be synchronized with the app information with reconciliation functionalities. First, as in the current app, when the patient scans new medicines to add to the list, the app needs to identify similar drugs and suggest potential duplicates. Scanning the packages should be a routine use of the app, to help keep correct counts of the countdown until refills are needed, and second to check for duplicates and perhaps also to detect drug interactions. Second, the patient can track the medications that are taken, which is a key information for the healthcare team, when it is available.

Although the medication list in the EPR is the list of prescribed (and delivered) medications, there may be differences with the patient's actual medication list: some doctors may not be connected to the patient's EPR, the patient may take over the counter drugs, and may actually have stopped taking some of the medications. This is why the patient needs to be able to edit his medication list without any restrictions (possible in the app). For example, during a phone consultation, the doctor may recommend a dose adjustment (e.g. "take half the pill instead of a full pill during the next 5 days") without changing the prescription, since the patient already has the medication. 


\section{Important Considerations for an Integrated Medication Management System}

Several existing barriers and anticipated obstacles need to be considered when implementing an integrated medication management system. An integrated system requires all stakeholders to have access to the system, and to use it regularly to have upto-date data. The first step is therefore to provide a secure access to the EPR for patients and providers, and to facilitate access to a free app for patients.

Patients will need an ID for the new EPR system to ensure a unique identity in Switzerland. The first challenge is that users of existing systems, such as our institutional patient portals and the medication app, do not yet rely on such an identifier. Therefore the existing identity and the new EPR ID must be reconciled.

Usability decreases accordingly with the efforts that are required for its use. The current EPR integrates high security standards that require health professionals to log into a system through a two-factor identification process. Since their usual electronic patient charts are not integrated yet, they will need to go through a double identification process with an SMS challenge for each patient chart. This is an interruptive, cumbersome process. With high clinical and administrative workload, these access issues may become an important barrier for regular use. The same issues exist for pharmacists.

The implementation of an integrated system must take into consideration the low or partial adoption of each tool in each part of the process. Individuals may adopt the EPR, but only use it occasionally to view their reports and prescriptions. Individuals may choose to only use the medication app, with or without sharing their information with their care-providers or with the hospital clinicians. The medication app is available in the app store, and will not be limited to individuals with EPR identification. Therefore, an ID reconciliation process with the unique ID must be anticipated for those who want to connect their chart to the EPR system after creating a personal login in the app.

The medication reconciliation process has been recognized as a key moment for patient safety, particularly during care transitions [5]. It needs to be carefully considered for each context:

- When admitting a patient to the hospital, the patient may have a different medication lists in the app and in the EPR. It is only after clarification with the patient that the hospital doctors can establish the list of current medications, because both sources may be discordant.

- When discharging the patient, the EPR medication list should be overridden by the hospital medication list. This list may be edited by the patient (who may stop some medications by themselves) or by the family doctor at the next visit.

- When the patient uses the app after discharge, the EPR medication list, annotated with the delivered medication list, will be available in the app. When scanning the medications, the patient can then check if there are doubles or similar medications from the prior medications at home (from before the hospitalization) that may need further clarification by the pharmacist or doctor.

We see that the reconciliation process is complex, particularly when the patient deals with medication at home. Even if the medication list may simply transit from the initial prescriber to the pharmacist, there is no way to guarantee correct information throughout the process due to high risk of undocumented changes or decisions from the patient (by both patient and care-providers). We must in particular be wary to avoid believing that what the doctor prescribes is what the patient actually takes at home, and allow the patient to correct and change the data as needed (orally or electronically). 


\section{Conclusion}

We conclude that an integrated medication system in Switzerland is feasible with the arrival of the new EPR system, and would further empower the patient for medication management. Besides having access to the EPR list, the availability of an app can help with daily adherence, medication safety (similar drugs and possible interactions) and allow the patient to edit the medication list without limitations. There are anticipated hurdles that need to be addressed, for access (identification and usability issues) and for the reconciliation process in each different situation.

\section{References}

[1] Peterson AM, Takiya LT, Finley R. Meta-analysis of trials of interventions to improve medication adherence. Am J Health Syst Pharm. 2003 Apr 1;60(7):657-65. doi: 10.1093/ajhp/60.7.657.

[2] Foppe van Mil JW, Westerlund T, Brown L, Chen TF, Henman M, et al. Medical care and drug-related problems: Do doctors and pharmacists speak the same language? Int J Clin Pharm. 2016;38(2):191-4.

[3] Viswanathan M, Golin CE, Jones CD, Ashok M, Blalock SJ, Wines RCM, Coker-Schwimmer EJL, Rosen DL, Sista P, Lohr KN. Interventions to Improve Adherence to Self-administered Medications for Chronic Diseases in the United States. Ann Intern Med [Internet]. 2012 Dec 4;157(11):785. Available from: http://annals.org/article.aspx?doi=10.7326/0003-4819-157-11-201212040-00538

[4] Davis MM, Devoe M, Kansagara D, Nicolaidis C, Englander H. "Did I do as best as the system would let me?" Healthcare professional views on hospital to home care transitions. J Gen Intern Med. 2012;27(12):1649-56.

[5] Redmond P, Grimes TC, Mcdonnell R, Boland F, Hughes C, Fahey T. Impact of medication reconciliation for improving transitions of care. Cochrane Database Syst Rev. 2018;2018(8).

[6] De Pietro C, Francetic I. E-health in Switzerland: The laborious adoption of the federal law on electronic health records (EHR) and health information exchange (HIE) networks. Health Policy (New York) [Internet]. 2018;122(2):69-74.

[7] Osborn CY, Mayberry LS, Wallston KA, Johnson KB, Elasy TA. Understanding patient portal use: Implications for medication management. J Med Internet Res. 2013;15(7).

[8] Ehrler F, Gschwind L, Meyer P, Blondon K. Swiss-Meds: An app fostering medication adherence of Swiss patient. Stud Health Technol Inform. 2019;259:71-6.

[9] Blondon K, Skalafouris C, Simonet ML, Piguet V. Using a Medication Plan as a Quality Indicator: Feasibility and Satisfaction Results from an Observational Study. Elzohry AAM, editor. J Fam Med [Internet]. 2019 Aug 24;1(3):15-23.

[10] Carpenter DM, Geryk LL, Chen AT, Nagler RH, Dieckmann NF, Han PKJ. Conflicting health information: a critical research need. Heal Expect. 2016;19(6):1173-82.

[11] Carpenter DM, Elstad EA, Blalock SJ, DeVellis RF. Conflicting Medication Information: Prevalence, Sources, and Relationship to Medication Adherence. J Health Commun [Internet]. 2014 Jan 9;19(1):6781.

[12] Bugnon B, Geissbuhler A, Bischoff T, Bonnabry P, von Plessen C. Improving primary care medication processes by using shared electronic medication plans in Switzerland: Lessons learned from a participatory action research study. JMIR Form Res. 2021;5(1):1-17.

[13] Park JYE, Li J, Howren A, Tsao NW, de Vera M. Mobile phone apps targeting medication adherence: Quality assessment and content analysis of user reviews. JMIR mHealth uHealth. 2019;7(1):1-12.

[14] Náfrádi L, Nakamoto K, Schulz PJ. Is patient empowerment the key to promote adherence? A systematic review of the relationship between self-efficacy, health locus of control and medication adherence. PLoS One. 2017;12(10):1-23.

[15] Ehrler F, Gschwind L, Meyer P, Blondon K. Swiss-Meds: An app fostering medication adherence of Swiss patient. Vol. 259, Studies in Health Technology and Informatics. 2019. 